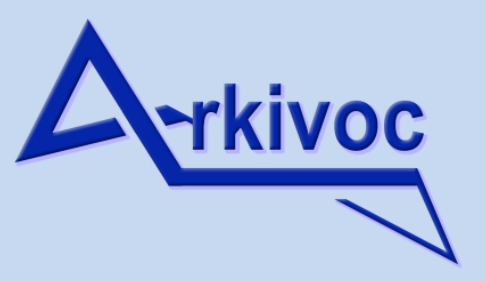

Free to Authors and Readers
A Platinum Open Access Journal for Organic Chemistry
Paper

Arkivoc 2022, part ii, 0-0

\title{
Synthesis of some oxazolo[4,5-d]pyrimidine derivatives and evaluation of their antiviral activity and cytotoxicity
}

Yevheniia Velihina, ${ }^{a}$ Stepan Pil'o, ${ }^{a}$ Oleksandr Kobzar, ${ }^{a}$ Olena Zaliavska, ${ }^{b}$ Mark N. Prichard, ${ }^{c^{* *}}$ Scott H. James, ${ }^{c}$ Kathy Keith, ${ }^{c}$ Caroll Hartline, ${ }^{c}$ Victor Zhirnov, ${ }^{a}$ Andriy Vovk, ${ }^{a}$ and Volodymyr Brovarets*a

${ }^{a}$ V.P. Kukhar Institute of Bioorganic Chemistry and Petrochemistry of the NAS of Ukraine, 1, Murmanska St, Kyiv 02094, Ukraine

${ }^{b}$ Department of Internal Medicine, Clinical Pharmacology and Occupational Diseases, Bukovinian State Medical University, 2, Sq. Teatralna, Chernivtsi 58002, Ukraine

'Department of Pediatrics, Division of Pediatric Infectious Diseases, University of Alabama at Birmingham, Birmingham, Alabama 35233, USA

Email: brovarets@bpci.kiev.ua

Dedicated to Prof. Girolamo Cirrincione in recognition of his outstanding contributions to the fields of organic and medicinal chemistry on the occasion of his retirement

Received 11-17-2021

Accepted Manuscript 01-24-2022

Published on line 02-03-2022

\section{Abstract}

New oxazolo[4,5- $d$ ]pyrimidine derivatives were synthesized and their some physicochemical and ADMET properties were analyzed by in silico prediction. Antiviral activity against DNA viruses (human cytomegalovirus, varicella-zoster virus, herpes simplex virus and BK virus) and cytotoxicity, including control experiments, were evaluated in vitro. Some of the compounds synthesized were characterized by low toxicity; however, they did not exhibit favorable antiviral effects.

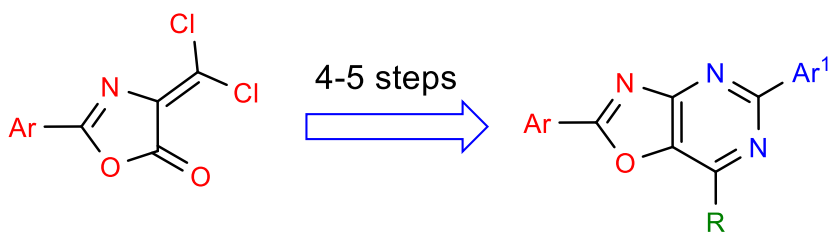

Antiviral evaluation

Cell toxicity

Pharmacokinetic properties

Keywords: Oxazolo[4,5- $d$ ] pyrimidine derivatives, synthesis, antiviral activity, cytotoxicity 


\section{Introduction}

Oxazole and pyrimidine scaffolds, as well as their fused heterocycles, are well-known structures utilized in the design of bioactive molecules in drug discovery. A significant number of studies have been carried out on the biological properties of oxazolo[5,4- $d]$ pyrimidines, uncovering their activities as receptor modulators and enzyme inhibitors. ${ }^{1}$ However, it seems that oxazolo[4,5-d]pyrimidines have only been synthesized, and that there is little information in the literature concerning their bioactivity. ${ }^{2}$ Among these bioactivity reports, inhibition of fatty acid amide hydrolase and monoglyceride lipase ${ }^{3}$ by these compounds have been described. Some of oxazolo[4,5- $d$ ] pyrimidine derivatives were also shown to exhibit anticancer activity. ${ }^{4-6}$ Since oxazoleand pyrimidine-based heterocycles, including oxazolo[5,4- $d]$ pyrimidines, have demonstrated antiviral effects, ${ }^{7-9}$ the synthesis of new compounds that contain oxazolo[4,5- $\left.d\right]$ pyrimidine fragment was of interest to us and therefore we decided to synthesize them and to evaluate their antiviral activity and toxicity.

In this paper, new oxazolo[4,5- $d$ ] pyrimidine derivatives were synthesized, characterized in silico on druglike properties and tested in vitro for cell cytotoxicity and efficacy against some viral opportunistic infections.

\section{Results and Discussion}

\section{Synthesis of target oxazolo[4,5-d]pyrimidines}

The synthesis of oxazolo[4,5-d]pyrimidines 1-9 is depicted in Scheme 1 and was carried out by a route described previously. ${ }^{4-6}$ Available oxazolones I were used as starting substrates. Oxazolo[4,5- $\left.d\right]$ pyrimidin$7(6 \mathrm{H})$-ones III were obtained through a simple sequence of the reactions I $\rightarrow$ II $\rightarrow$ III (Scheme 1). ${ }^{10}$ The chlorination of these compounds $\left(\mathrm{POCl}_{3}\right.$ (excess), $\mathrm{Me}_{2} \mathrm{NPh}, 105-110^{\circ} \mathrm{C}$ ) afforded 7-chlorosubstituted oxazolo[4,5- $d$ ] pyrimidines IV. ${ }^{4-6,10}$ Treatment of compounds IV with the primary or secondary amines, in the presence of triethylamine in dioxane, resulted in high yields of the corresponding 7-aminesubstituted oxazolo[4,5- $d$ ] pyrimidines 1-9. The synthesis of oxazolo[4,5- $d$ ]pyrimidines $\mathbf{V}$ was accomplished in analogy to previously reported compounds. ${ }^{4-6}$

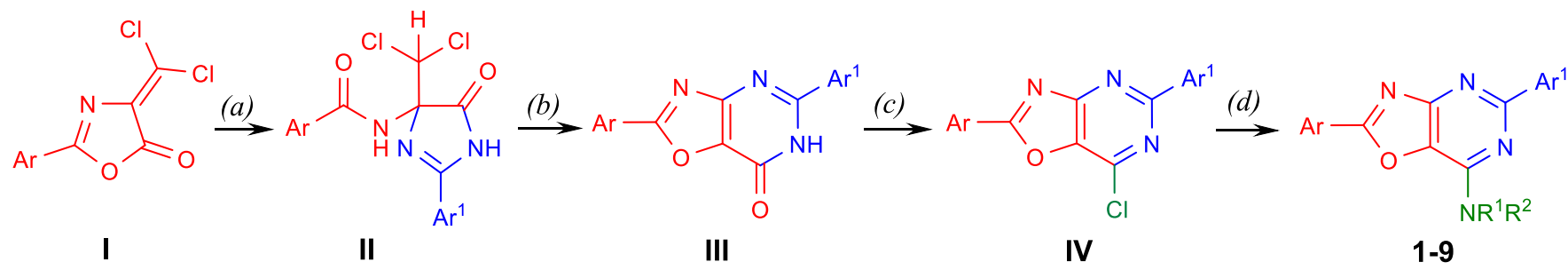

Scheme 1. Synthesis of oxazolo[4,5- $d$ ]pyrimidines 1-15. Reagents and conditions: $(a)$ amidine hydrochlorides, $\mathrm{Et}_{3} \mathrm{~N}$, THF, r.t., 48h; (b) Py, reflux, $10 \mathrm{~h} ;(c) \mathrm{POCl}_{3}, \mathrm{Me}_{2} \mathrm{NPh}, 105-110{ }^{\circ} \mathrm{C}$, 3h; (d) corresponding amine, dioxane, reflux, $6 h$.

The treatment of derivatives $\mathbf{V}$ with alkyl- or arylsulfonyl chlorides, in the presence of triethylamine and on heating in dioxane, led to new oxazolo[4,5-d]pyrimidines 10-15 (Scheme 2). The composition and structure of compounds 1-15 were confirmed by elemental analysis, ${ }^{1} \mathrm{H}$ and ${ }^{13} \mathrm{C}$ NMR spectra and LC-MS spectrometry.

The synthesized compounds 1-15 (Figure 1) are represented by 5-(4-methylphenyl)-2-phenyl-7substituted oxazolo[4,5-d] pyrimidine derivatives 1-9, 2-(4-methylphenyl)-5-phenyl-7-substituted oxazolo[4,5- 
d]pyrimidine derivatives 10-12, 14, 15, and 2,5-diphenyl-7-substituted oxazolo[4,5-d]pyrimidine 13. The physicochemical properties of the molecules were modulated by the nature of the substituent at position 7 .

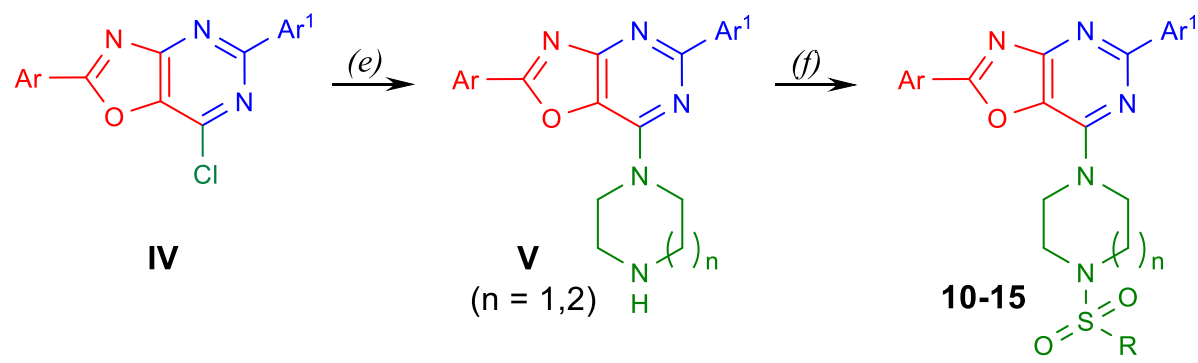

Scheme 2. Synthesis of oxazolo[4,5-d]pyrimidines 10-15. Reagents and conditions: (e) piperazine or 1,4diazepane, dioxane, reflux, $6 \mathrm{~h}$; $(f)$ corresponding $\mathrm{RSO}_{2} \mathrm{Cl}_{\text {, }} \mathrm{Et}_{3} \mathrm{~N}$, dioxane, $105-110{ }^{\circ} \mathrm{C}, 6 \mathrm{~h}$.

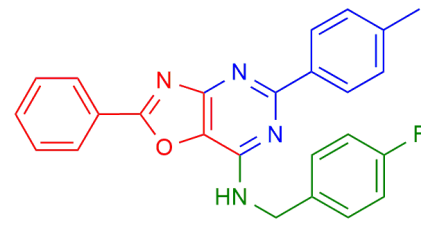

1

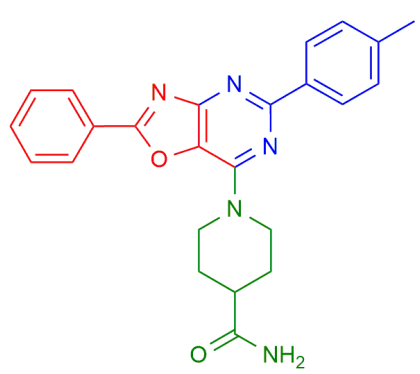

5

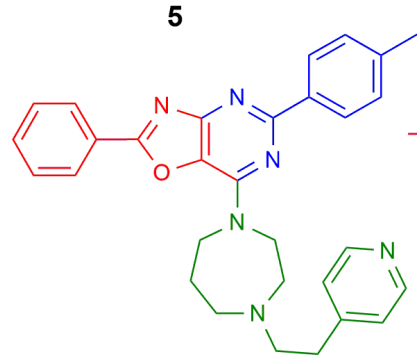

9

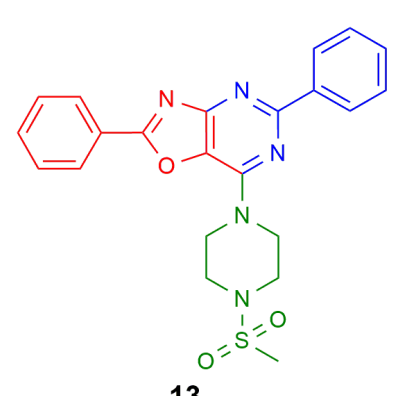

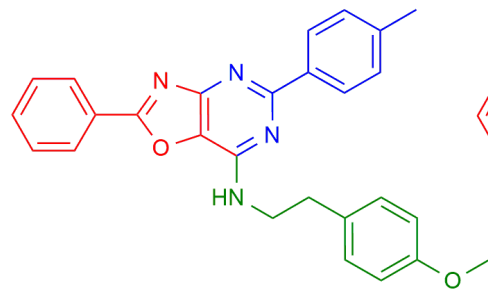

2

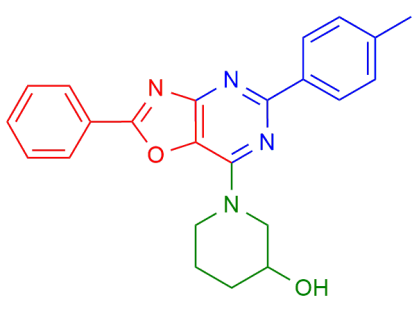

6

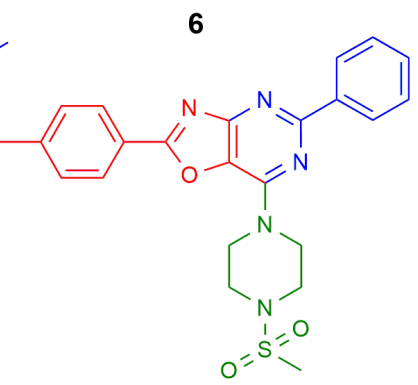

10

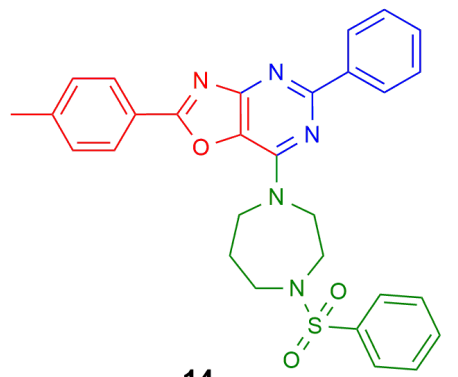

14
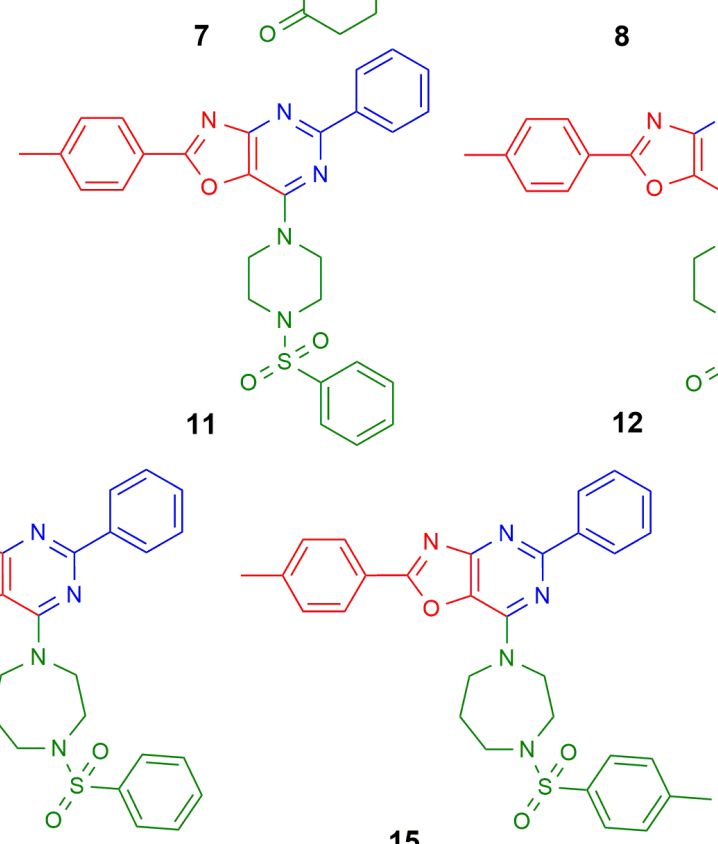
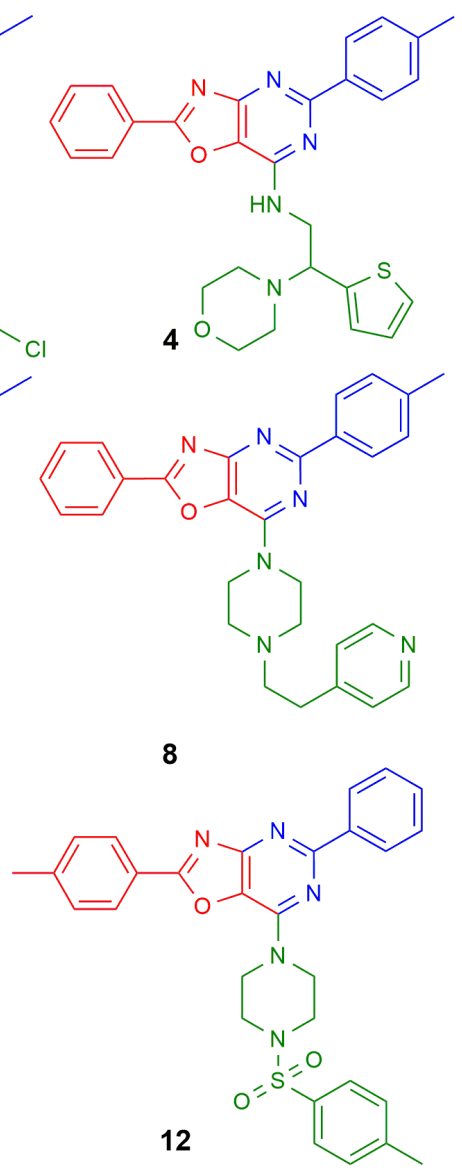

15

Figure 1. Synthesized oxazolo[4,5-d]pyrimidine derivatives 1-15. 


\section{Investigation of molecular properties and drug-like parameters}

Drug-like parameters (Table 1), as calculated by Molinspiration software (http://www.molinspiration.com), indicated that only four of the 15 synthesized compounds are in compliance with Lipinski rule of five. ${ }^{11}$ Twothirds of the derivatives have molecular weights lower than $500 \mathrm{Da}$. Accordig to the predictive software, only compounds 5, 6, 10, and $\mathbf{1 3}$ are expected to have $\log P$ values lower than 5 , and the other oxazolo[4,5d]pyrimidine derivatives are more lipophilic. All of the compounds were predicted to have $\mathrm{H}$-bond accepting abilities in the range of 5-8, and less than half of them can be characterized by one $\mathrm{H}$-bond donating group. The topological polar surface area prediction values (from 64 to 94) were found to be in the acceptable range. Pharmacokinetic properties (Table 1 and Table S1) predicted by the pkCSM online server ${ }^{12}$ suggested that all the oxazolo[4,5-d]pyrimidine derivatives could potentially have good human intestinal absorption, and compound 1 can also have a good possibility in terms of blood-brain barrier permeability.

Table 1. Some physicochemical parameters and pharmacokinetic properties of oxazolo[4,5- $d$ ]pyrimidines 1-15

\begin{tabular}{ccccccccc}
\hline Compd & $\begin{array}{c}\text { Molecular } \\
\text { weight }\end{array}$ & milogP & nOHNH & nON & nrotb & $\begin{array}{c}\text { TPSA }^{\mathrm{e}}, \\
\AA^{2}\end{array}$ & $\begin{array}{c}\text { Intestinal } \\
\text { absorption } \\
\text { (human), } \%\end{array}$ & $\begin{array}{c}\text { BBB } \\
\text { permeability, } \\
\text { logBB }\end{array}$ \\
\hline $\mathbf{1}$ & 410.45 & 6.15 & 1 & 5 & 5 & 63.84 & 93.366 & 0.701 \\
$\mathbf{2}$ & 436.51 & 6.45 & 1 & 6 & 7 & 73.08 & 91.915 & -0.393 \\
$\mathbf{3}$ & 526.04 & 6.72 & 1 & 7 & 7 & 76.31 & 92.473 & -0.370 \\
$\mathbf{4}$ & 497.62 & 5.94 & 1 & 7 & 7 & 76.31 & 92.322 & -0.405 \\
$\mathbf{5}$ & 413.48 & 4.34 & 2 & 7 & 4 & 98.15 & 97.038 & -0.862 \\
$\mathbf{6}$ & 386.45 & 4.59 & 1 & 6 & 3 & 75.28 & 96.449 & -0.640 \\
$\mathbf{7}$ & 424.50 & 5.28 & 0 & 6 & 3 & 72.12 & 95.794 & -0.697 \\
$\mathbf{8}$ & 476.58 & 5.24 & 0 & 7 & 6 & 71.18 & 97.391 & -0.861 \\
$\mathbf{9}$ & 490.61 & 5.51 & 0 & 7 & 6 & 71.18 & 96.989 & -0.882 \\
$\mathbf{1 0}$ & 449.54 & 4.10 & 0 & 8 & 4 & 92.43 & 95.375 & -1.209 \\
$\mathbf{1 1}$ & 511.61 & 5.63 & 0 & 8 & 5 & 92.43 & 100 & -1.245 \\
$\mathbf{1 2}$ & 525.63 & 6.08 & 0 & 8 & 5 & 92.43 & 100 & -1.255 \\
$\mathbf{1 3}$ & 435.51 & 3.65 & 0 & 8 & 4 & 92.43 & 95.809 & -1.231 \\
$\mathbf{1 4}$ & 525.63 & 5.90 & 0 & 8 & 5 & 92.43 & 100 & -1.240 \\
$\mathbf{1 5}$ & 539.66 & 6.35 & 0 & 8 & 5 & 92.43 & 100 & -1.250 \\
\hline
\end{tabular}

${ }^{a}$ miLogP - LogP prediction method developed at Molinspiration. ${ }^{b} \mathrm{nOHNH}-\mathrm{Number}$ of $\mathrm{OH}$ and $\mathrm{NH}$ groups as $\mathrm{H}$-bond donors. ${ }^{\mathrm{C} O \mathrm{~N}}-\mathrm{Number}$ of $\mathrm{O}$ and $\mathrm{N}$ atoms as $\mathrm{H}$-bond acceptors. ${ }^{\mathrm{d}}$ nrotb - Number of rotatable bonds. ${ }^{\text {eTPSA }}$ - Topological polar surface area.

\section{Antiviral and Cytotoxicity Assays}

Activity against human cytomegalovirus (HCMV), varicella-zoster virus (VZV), herpes simplex virus (HSV-1) and BK virus (BKV) was assessed via in vitro antiviral assays. The data obtained in the study, along with positive controls for each virus, are given in Table 2. Only compound 3 showed activity against BKV with EC 50 of 3.51 $\mu \mathrm{M}$ and $\mathrm{CC}_{50}$ of $23.53 \mu \mathrm{M}$ (selectivity index $\mathrm{SI}_{50}$ is 7 ), and none of the other compounds demonstrated favorable antiviral activity. At the same time, $\mathrm{CC}_{50}$ values indicated a high degree of cytotoxicity of many of the tested compounds. It should also be noted, that in silico ADMET profile showed that the compounds could potentially inhibit hERG II and exhibit hepatotoxicity (Table S1). 
Table 2. In vitro antiviral activity and cell cytotoxicity of oxazolo[4,5-d]pyrimidine derivatives 1-15 in primary assay

\begin{tabular}{ccccccccc}
\hline \multirow{2}{*}{ Compd } & \multicolumn{2}{c}{$\mathrm{HCMV}^{\mathrm{a}}$} & \multicolumn{2}{c}{$\mathrm{VZV}^{\mathrm{b}}$} & \multicolumn{2}{c}{$\mathrm{BKV}^{\mathrm{c}}$} & \multicolumn{2}{c}{$\mathrm{HSV}^{\mathrm{d}}$} \\
\cline { 2 - 9 } $\mathbf{1}$ & $\mathrm{EC} 50$ & $\mathrm{CC}_{50}$ & $\mathrm{EC}_{50}$ & $\mathrm{CC}_{50}$ & $\mathrm{EC}_{50}$ & $\mathrm{CC}_{50}$ & $\mathrm{EC}_{50}$ & $\mathrm{CC}_{50}$ \\
\hline $\mathbf{2}$ & $>1.00$ & 13.79 & $>1.20$ & 3.76 & $\mathrm{ND}$ & $\mathrm{ND}$ & $>1.20$ & 4.04 \\
$\mathbf{3}$ & $>6.00$ & 20.63 & $>30.00$ & 37.54 & 3.51 & 23.53 & $>6.00$ & 19.62 \\
$\mathbf{4}$ & $>1.20$ & 5.82 & $>6.00$ & 21.47 & 15.12 & 37.24 & $>6.00$ & 17.53 \\
$\mathbf{5}$ & $>150.00$ & $>150.00$ & $>150.00$ & $>150.00$ & $\mathrm{ND}$ & $\mathrm{ND}$ & $>150.00$ & $>150.00$ \\
$\mathbf{6}$ & $>150.00$ & $>150.00$ & 62.07 & $>150.00$ & $\mathrm{ND}$ & $\mathrm{ND}$ & $>150.00$ & $>150.00$ \\
$\mathbf{7}$ & $>30.00$ & 91.42 & $>30.00$ & 87.35 & $\mathrm{ND}$ & $\mathrm{ND}$ & $>30.00$ & 77.65 \\
$\mathbf{8}$ & $>150.00$ & $>150.00$ & $>150.00$ & $>150.00$ & $>150.00$ & $>150.00$ & $>30.00$ & 110.00 \\
$\mathbf{9}$ & $>1.20$ & 3.14 & $>1.20$ & 3.64 & $>1.20$ & 5.32 & $>1.20$ & 2.96 \\
$\mathbf{1 0}$ & 114.11 & $>150.00$ & 139.62 & $>150.00$ & $>150.00$ & $>150.00$ & $>150.00$ & $>150.00$ \\
$\mathbf{1 1}$ & $>30.00$ & 88.41 & $>30.00$ & 73.83 & $>150.00$ & $>150.00$ & $>30.00$ & 78.34 \\
$\mathbf{1 2}$ & $>30.00$ & 96.24 & $>6.00$ & 16.94 & $>30.00$ & 86.07 & $>6.00$ & 15.92 \\
13 & $>150.00$ & $>150.00$ & 81.07 & $>150.00$ & 119.82 & $>150.00$ & $>150.00$ & $>150.00$ \\
$\mathbf{1 4}$ & $>30.00$ & 105.32 & $>30.00$ & 100.07 & $>30.00$ & 93.31 & $>30.00$ & 117.00 \\
$\mathbf{1 5}$ & $>1.20$ & 3.05 & $>1.20$ & 3.16 & $>1.20$ & 5.27 & $>1.20$ & 3.32 \\
\hline
\end{tabular}

Positive controls: a Ganciclovir: 1.10, 0.68, 0.94 (EC 50$)$; >150.00 ( $\left.\mathrm{CC}_{50}\right)$; ${ }^{\mathrm{b}}$ Acyclovir: 3.28, 2.25 (EC 50$)$; $>150.00$ (CC50); ' Cidofovir: 0.42 (EC50); >100.00 (CC 50$)$; ${ }^{\mathrm{d} A c y c l o v i r: ~ 0.72, ~} 0.78$ (EC 50$)$; >150.00 (CC 50$)$. eND - not determined.

Giving the in vitro cytotoxicity results, we analyzed the possible link between the toxicity of the tested compounds and their lipophilicity. Cytotoxic compounds 1-4, 9, and 15 have calculated logP values from 5.51 to 6.72 , while the compounds with low toxicity $\left(C_{50}>150 \mu \mathrm{M}\right), \mathbf{5}, \mathbf{6}, \mathbf{8}, \mathbf{1 0}, \mathbf{1 3}$, are characterized by logP values from 3.65 to 5.24 . This indicates that high lipophilicity of the oxazolo[4,5- $d$ ]pyrimidine derivatives may cause their high toxicity, probably due to higher permeability through the membrane and binding to intracellular targets of both infected and non-infected cells.

\section{Conclusions}

New oxazolo[4,5- $d$ ]pyrimidine derivatives were synthesized and their antiviral activities against four DNA viruses were evaluated in vitro. Some of physicochemical and pharmacological properties of the compounds were predicted in silico. Biologically relevant antiviral assays demonstrated a lack of antiviral effects of the compounds, with cytotoxicity being a major limitation in many of them. This could be due to the high predicted logP values for the compounds and is consistent with predicted good human intestinal absorption. Therefore, the main task of further functionalization of the oxazolo[4,5- $d$ ]pyrimidine framework is to reduce their toxicity and increase the selectivity. 


\section{Experimental Section}

General. All chemicals and solvents for the synthetic work were acquired from commercial sources and used without further purification. Reaction monitoring by thin layer chromatography was performed on pre-coated alumina plates $\mathrm{SiO}_{2} 60 \mathrm{~F}_{254}$ from Merck. Melting points were determined on a Fisher-Johns apparatus. ${ }^{1} \mathrm{H}$ and ${ }^{13} \mathrm{C}$ NMR spectra were recorded on Bruker Avance DRX 500 or Varian Mercury 400 spectrometers in DMSO- $d_{6}$ or $\mathrm{CDCl}_{3}$ taking its residual protons signal as a standard. Data are reported as follows: chemical shift, multiplicity ( $s=$ singlet, $d=$ doublet, $t=$ triplet, $q=$ quartet, $m=$ multiplet), integration, and assignment. Broad signals are indicated as br. Coupling constants $(J)$ are given in Hertz [Hz]. LC-MS identification was conducted on an Agilent 1200 Series system equipped with a diode array and a G6130A mass-spectrometer (atmospheric pressure electrospray ionization). Combustion elemental analysis was performed by hand in the V.P. Kukhar Institute of Bioorganic Chemistry and Petrochemistry analytical laboratory. The Carbon and Hydrogen contents were determined using the Pregl gravimetric method, Nitrogen - using the Duma's gasometrical micromethod, Sulfur - by the Scheininger titrimetric method, Chlorine - by the mercurometric method. Oxazolo[4,5- $d$ ] pyrimidines IV, V were synthesized according to previously described methods. ${ }^{4,5}$

General procedure for the synthesis of 7-aminesubstituted oxazolo[4,5-d]pyrimidines 1-9. A mixture of compound IV $(2 \mathrm{mmol})$, one of amines $(2 \mathrm{mmol})$ and $\mathrm{Et}_{3} \mathrm{~N}(2 \mathrm{mmol})$ in dioxane $(10 \mathrm{~mL})$ was refluxed for $6 \mathrm{~h}$. After removal of the solvent, the residue was triturated with water, filtered off, dried and recrystallized from mixture of acetonitrile and dimethylformamide (5:1) obtaining white solids that are described below.

$\boldsymbol{N}$-(4-Fluorobenzyl)-5-(4-methylphenyl)-2-phenyl-oxazolo[4,5-d]pyrimidin-7-amine (1). Yield: 80\%. mp 190 $192{ }^{\circ} \mathrm{C} .{ }^{1} \mathrm{H}-\mathrm{NMR}\left(400 \mathrm{MHz}, \mathrm{DMSO}-d_{6}\right): \delta 8.76(s, 1 \mathrm{H}, \mathrm{H}-\mathrm{N}), 8.26(d, J 8.0 \mathrm{~Hz}, 2 \mathrm{H}, \mathrm{ArH}), 8.21(d, J 6.8 \mathrm{~Hz}, 2 \mathrm{H}, \mathrm{ArH})$, $7.71-7.63(m, 3 \mathrm{H}, \mathrm{ArH}), 7.54-7.49(m, 2 \mathrm{H}, \mathrm{ArH}), 7.28(d, J 8.0 \mathrm{~Hz}, 2 \mathrm{H}, \mathrm{ArH}), 7.17(t, J 8.9 \mathrm{~Hz}, 2 \mathrm{H}, \operatorname{ArH}), 4.84(d$, J $\left.6.0 \mathrm{~Hz}, 2 \mathrm{H}, \mathrm{CH}_{2}\right), 2.36$ (s, 3H, Me). ${ }^{13} \mathrm{C}-\mathrm{NMR}\left(101 \mathrm{MHz}, \mathrm{DMSO}-d_{6}\right): \delta 164.3,162.5,160.1,159.9,139.6,136.5$, 135.8, 135.3, 132.7, 129.8, 129.4, 129.0, 127.8, 127.5, 126.0, 115.2, 115.0, 42.8, 21.0. MS: $411.0\left([M+]^{+}\right.$, $\mathrm{C}_{25} \mathrm{H}_{20} \mathrm{FN}_{4} \mathrm{O}^{+}$, calc. 411.2). Anal. calc. for $\mathrm{C}_{25} \mathrm{H}_{19} \mathrm{FN}_{4} \mathrm{O}$ (410.15): C 73.16, $\mathrm{H} 4.67, \mathrm{~N}$ 13.65, found: $\mathrm{C} 73.13, \mathrm{H} 4.65$, N 13.58.

N-(4-Methoxyphenethyl)-5-(4-methylphenyl)-2-phenyl-oxazolo[4,5-d]pyrimidin-7-amine (2). Yield: 82\%. mp $151-153^{\circ} \mathrm{C} .{ }^{1} \mathrm{H}-\mathrm{NMR}\left(400 \mathrm{MHz}, \mathrm{DMSO}-d_{6}\right): \delta 8.29$ (br d, J 7.9 Hz, 3H, ArH, NH), $8.23-8.20(\mathrm{~m}, 2 \mathrm{H}, \mathrm{ArH}), 7.72$ $-7.65(\mathrm{~m}, 3 \mathrm{H}, \mathrm{ArH}), 7.31$ (d, J $7.9 \mathrm{~Hz}, 2 \mathrm{H}, \mathrm{ArH}), 7.26-7.23(\mathrm{~m}, 2 \mathrm{H}, \mathrm{ArH}), 6.87$ (d, J $8.0 \mathrm{~Hz}, 2 \mathrm{H}, \mathrm{ArH}), 3.84-3.80$ $\left(\mathrm{m}, 2 \mathrm{H}, \mathrm{CH}_{2}\right), 3.71(\mathrm{~s}, 3 \mathrm{H}, \mathrm{MeO}), 2.96\left(\mathrm{t}, J 7.5 \mathrm{~Hz}, 2 \mathrm{H}, \mathrm{CH}_{2}\right), 2.38(\mathrm{~s}, 3 \mathrm{H}, \mathrm{Me}) .{ }^{13} \mathrm{C}-\mathrm{NMR}\left(126 \mathrm{MHz}, \mathrm{DMSO}-d_{6}\right): \delta$ 131.6, 129.0, 128.2, 127.5, 127.2, 125.4, 113.3, 54.4, 20.6. MS: $437.0\left([M+\mathrm{H}]^{+}, \mathrm{C}_{27} \mathrm{H}_{25} \mathrm{FN}_{4} \mathrm{O}_{2}{ }^{+}\right.$, calc. 437.2). Anal. calc. for $\mathrm{C}_{27} \mathrm{H}_{24} \mathrm{~N}_{4} \mathrm{O}_{2}$ (436.19): C 74.29, $\mathrm{H} 5.54, \mathrm{~N} 12.84$, found: C 74.30, H 5.50, N 12.78 .

\section{N-(2-(4-Chlorophenyl)-2-morpholinoethyl)-5-(4-methoxyphenethyl)-2-phenyl-oxazolo[4,5-d]pyrimidin-7-}

amine (3). Yield: $78 \%$. mp $225-227^{\circ} \mathrm{C} .{ }^{1} \mathrm{H}-\mathrm{NMR}\left(400 \mathrm{MHz}, \mathrm{DMSO}-d_{6}\right): \delta 8.29-8.10(m, 5 \mathrm{H}, \mathrm{ArH}), 7.71-7.65$ ( $m, 3 \mathrm{H}, \mathrm{ArH}), 7.37$ (br s, 4H, ArH), $7.29(d, J 7.8 \mathrm{~Hz}, 2 \mathrm{H}, \mathrm{ArH}), 4.26-4.23(m, 1 \mathrm{H}, \mathrm{CH}), 3.92-3.84\left(m, 2 \mathrm{H}, \mathrm{CH}_{2}\right)$, $3.56-3.54\left(m, 4 \mathrm{H}, \mathrm{CH}_{2}\right), 2.45$ (br s, $\left.4 \mathrm{H}, \mathrm{CH}_{2}\right), 2.37$ (s, 3H, Me). ${ }^{13} \mathrm{C}-\mathrm{NMR}\left(126 \mathrm{MHz}^{\mathrm{CDCl}} \mathrm{CD}_{3}\right): \delta$ 161.4, 147.9, 140.4, 135.4, 132.6, 130.2, 129.2, 128.4, 128.2, 126.5, 120.0, 50.7, 21.6. Anal. calc. for $\mathrm{C}_{30} \mathrm{H}_{28} \mathrm{~N}_{4} \mathrm{O}_{2}$ (525.19): C 68.50, H 5.37, Cl 6.74, N 13.31, found: C 68.48, H 5.40, Cl 6.68, N 13.25.

$\mathbf{N}$-(2-Morpholino-2-(thiophen-2-yl)ethyl)-5-(4-methoxyphenethyl)-2-phenyl-oxazolo[4,5-d]pyrimidin-7amine (4). Yield: $75 \%$. mp $226-228{ }^{\circ} \mathrm{C} .{ }^{1} \mathrm{H}-\mathrm{NMR}\left(400 \mathrm{MHz}, \mathrm{DMSO}-d_{6}\right): \delta 8.28-8.26(m, 3 \mathrm{H}, \mathrm{ArH}, \mathrm{NH}), 8.20(b r$ $d, J 7.1 \mathrm{~Hz}, 2 \mathrm{H}, \mathrm{ArH}), 7.70-7.63(m, 3 \mathrm{H}, \mathrm{ArH}), 7.49-7.47(m, 1 \mathrm{H}, \mathrm{HetH}), 7.29(d, J 7.9 \mathrm{~Hz}, 2 \mathrm{H}, \mathrm{ArH}), 7.10(d, J$ $3.5 \mathrm{~Hz}, 1 \mathrm{H}, \mathrm{HetH}), 7.06-7.04(m, 1 \mathrm{H}, \mathrm{HetH}), 4.33(t, J 6.9 \mathrm{~Hz}, 1 \mathrm{H}, \mathrm{CH}), 4.22-4.16(m, 1 \mathrm{H}, \mathrm{CH}), 4.05-4.03(m$, $1 \mathrm{H}, \mathrm{CH}), 3.97-3.94(m, 1 \mathrm{H}, \mathrm{CH}), 3.83(t, J 4.7 \mathrm{~Hz}, 1 \mathrm{H}, \mathrm{CH}), 3.55\left(\right.$ br s, $\left.2 \mathrm{H}, \mathrm{CH}_{2}\right), 2.58\left(\right.$ br s, $\left.2 \mathrm{H}, \mathrm{CH}_{2}\right), 2.37(s, 3 \mathrm{H}$, 
Me). ${ }^{13} \mathrm{C}-\mathrm{NMR}\left(126 \mathrm{MHz}\right.$, DMSO-d $\left.d_{6}\right): \delta$ 159.9, 154.8, 139.6, 132.7, 129.4, 129.0, 127.8, 127.7, 127.6, 126.7, 125.2, 66.5, 66.0, 49.5, 21.0. Anal. calc. for $\mathrm{C}_{28} \mathrm{H}_{27} \mathrm{~N}_{5} \mathrm{O}_{2} \mathrm{~S}$ (497.19): C 67.58, H 5.47, N 14.07, S 6.44, found: C $67.60, \mathrm{H} 5.51, \mathrm{~N} 14.10, \mathrm{~S} 6.48$.

1-(5-(4-Methylphenyl)-2-phenyl-oxazolo[4,5-d]pyrimidin-7-yl)piperidine-4-carboxamide (5). Yield: 70\%. $\mathrm{mp}$ $\left.299-301{ }^{\circ} \mathrm{C} .{ }^{1} \mathrm{H}-\mathrm{NMR}(400 \mathrm{MHz} \text {, DMSO-d })_{6}\right): \delta 8.28-8.24(m, 4 \mathrm{H}, \mathrm{ArH}), 7.73-7.64(m, 3 \mathrm{H}, \mathrm{ArH}), 7.30(\mathrm{~d}, J 7.9 \mathrm{~Hz}$, $3 \mathrm{H}, \mathrm{ArH}, \mathrm{NH}_{2}$ ), 6.79 (br s, 1H, $\mathrm{NH}_{2}$ ), 4.84 (d, J $13.4 \mathrm{~Hz}, 2 \mathrm{H}, \mathrm{CH}_{2}$ ), 3.34 (br s, 2H, CH $\left.13.1 \mathrm{~Hz}, 2 \mathrm{H}, \mathrm{CH}_{2}\right), 1.74-1.66\left(\mathrm{~m}, 2 \mathrm{H}, \mathrm{CH}_{2}\right) \cdot{ }^{13} \mathrm{C}-\mathrm{NMR}\left(126 \mathrm{MHz}, \mathrm{DMSO}-d_{6}\right): \delta$ 164.5, 162.6, 159.8, 139.8, 135.4, 132.8, 129.4, 129.0, 127.8, 28.4, 21.0. MS: $414.2\left([\mathrm{M}+\mathrm{H}]^{+}, \mathrm{C}_{24} \mathrm{H}_{24} \mathrm{~N}_{5} \mathrm{O}_{2}{ }^{+}\right.$, calc. 414.2). Anal. calc. for $\mathrm{C}_{24} \mathrm{H}_{23} \mathrm{~N}_{5} \mathrm{O}_{2}$ (413.19): C 69.72, H 5.61, N 16.94, found: C 69.70, H 5.63, N 17.00.

1-(5-(4-Methylphenyl)-2-phenyl-oxazolo[4,5-d]pyrimidin-7-yl)piperidin-3-ol (6). Yield: 72\%. mp $291-293{ }^{\circ} \mathrm{C}$. ${ }^{1} \mathrm{H}-\mathrm{NMR}\left(400 \mathrm{MHz}, \mathrm{DMSO}-d_{6}\right): \delta 8.26-8.22(m, 4 \mathrm{H}, \mathrm{ArH}), 7.72-7.62(m, 3 \mathrm{H}, \mathrm{ArH}), 7.28(d, J 8.0 \mathrm{~Hz}, 2 \mathrm{H}, \mathrm{ArH})$, $5.06(d, J 4.2 \mathrm{~Hz}, 1 \mathrm{H}, \mathrm{OH}), 4.47(b r s, 1 \mathrm{H}, \mathrm{CH}), 4.28($ br d, J $13.2 \mathrm{~Hz}, 1 \mathrm{H}, \mathrm{CH}), 3.71($ br s, $1 \mathrm{H}, \mathrm{CH}), 3.64-3.58(m$, $1 \mathrm{H}, \mathrm{CH}), 3.42(b r s, 1 \mathrm{H}, \mathrm{CH}), 2.36(s, 3 \mathrm{H}, \mathrm{Me}), 1.97-1.86\left(m, 2 \mathrm{H}, \mathrm{CH}_{2}\right), 1.61-1.51\left(m, 2 \mathrm{H}, \mathrm{CH}_{2}\right) .{ }^{13} \mathrm{C}-\mathrm{NMR}(126$ $\mathrm{MHz}$, DMSO- $\left.d_{6}\right): \delta 163.9,161.5,159.4,147.8,139.6,135.2,132.7,129.4,128.9,127.7,127.7,125.7,65.0,32.7$, 22.4, 21.0. MS: $387.0\left([\mathrm{M}+\mathrm{H}]^{+}, \mathrm{C}_{23} \mathrm{H}_{23} \mathrm{~N}_{4} \mathrm{O}_{2}{ }^{+}\right.$, calc. 387.2). Anal. calc. for $\mathrm{C}_{23} \mathrm{H}_{22} \mathrm{~N}_{4} \mathrm{O}_{2}$ (386.17): C 71.48, $\mathrm{H}$ 5.74, $\mathrm{N}$ 14.50, found: C 71.46, H 5.70, N 14.56 .

5-(4-Methylphenyl)-3-(2-phenyl-oxazolo[4,5-d]pyrimidin-7-yl)-1,2,3,4,5,6-hexahydro-8H-1,5-methanopyrido[1,2-a][1,5]diazocin-8-one (7). Yield: $78 \%$. mp $263-265{ }^{\circ} \mathrm{C} .{ }^{1} \mathrm{H}-\mathrm{NMR}\left(400 \mathrm{MHz}, \mathrm{DMSO}-d_{6}\right): \delta 8.30-8.23$ $(m, 2 \mathrm{H}, \mathrm{ArH}), 8.20-8.17(m, 2 \mathrm{H}, \mathrm{ArH}), 7.75-7.62(m, 3 \mathrm{H}, \mathrm{ArH}), 7.28(d, J 8.0 \mathrm{~Hz}, 2, \mathrm{ArH} \mathrm{H}), 7.04(b r s, 1 \mathrm{H}$, HetH), $6.34(d, J 6.9 \mathrm{~Hz}, 1 \mathrm{H}, \mathrm{HetH}), 5.90(d, J 9.0 \mathrm{~Hz}, 1 \mathrm{H}, \mathrm{HetH}), 5.12($ br s, $1 \mathrm{H}, \mathrm{CH}), 4.88(b r s, 1 \mathrm{H}, \mathrm{CH}), 4.03(d, J$ $15.6 \mathrm{~Hz}, 1 \mathrm{H}, \mathrm{CH}), 3.70-3.40\left(m, 4 \mathrm{H}, \mathrm{CH}_{2}\right), 2.68(b r s, 1 \mathrm{H}, \mathrm{CH}), 2.37(s, 3 \mathrm{H}, \mathrm{Me}), 2.18(b r d, J 12.9 \mathrm{~Hz}, 1 \mathrm{H}, \mathrm{CH})$, 2.07 (br d, J $12.7 \mathrm{~Hz}, 1 \mathrm{H}, \mathrm{CH}) .{ }^{13} \mathrm{C}-\mathrm{NMR}(126 \mathrm{MHz}$, DMSO-d $): \delta 164.1,161.8,161.7,159.2,157.5,147.9,146.1$, 139.7, 138.5, 135.0, 132.9, 129.4, 128.9, 127.7, 127.0, 125.5, 115.7, 104.8, 48.5, 34.3, 27.5, 25.6, 21.0. MS: $476.2\left([\mathrm{M}+\mathrm{H}]^{+}, \mathrm{C}_{29} \mathrm{H}_{26} \mathrm{~N}_{5} \mathrm{O}_{2}{ }^{+}\right.$, calc. 476.2). Anal. calc. for $\mathrm{C}_{29} \mathrm{H}_{25} \mathrm{~N}_{5} \mathrm{O}_{2}$ (475.20): C 73.25, H 5.30, N 14.73, found: C 73.22, H 5.28, N 14.74 .

5-(4-Methylphenyl)-2-phenyl-7-(4-(2-(pyridin-4-yl)ethyl)piperazin-1-yl)-oxazolo[4,5-d]pyrimidine (8). Yield: 86\%. mp $188-190{ }^{\circ} \mathrm{C} .{ }^{1} \mathrm{H}-\mathrm{NMR}\left(400 \mathrm{MHz}\right.$, DMSO- $\left.d_{6}\right): \delta 8.47-8.46(m, 2 \mathrm{H}, \mathrm{ArH}), 8.27-8.24(m, 4 \mathrm{H}, \mathrm{ArH}), 7.72$ $-7.63(m, 3 \mathrm{H}, \mathrm{ArH}), 7.32-7.28(m, 4 \mathrm{H}, \mathrm{ArH}), 4.04\left(b r s, 4 \mathrm{H}, \mathrm{CH}_{2}\right), 2.83\left(t, J 7.5 \mathrm{~Hz}, 2 \mathrm{H}, \mathrm{CH}_{2}\right), 2.67-2.64(m, 6 \mathrm{H}$, $\left.\mathrm{CH}_{2}\right), 2.37(s, 3 \mathrm{H}, \mathrm{Me}) .{ }^{13} \mathrm{C}$ NMR $\left(126 \mathrm{MHz}, \mathrm{DMSO}-d_{6}\right): \delta 164.2,161.7,159.5,152.4,149.4,147.7,139.7,135.1$, 132.8, 129.4, 129.0, 127.7, 125.6, 124.3, 58.1, 52.3, 31.7, 21.0. MS: $477.2\left(\left[M+\mathrm{H}^{+}, \mathrm{C}_{29} \mathrm{H}_{29} \mathrm{~N}_{6} \mathrm{O}^{+}\right.\right.$, calc. 477.2). Anal. calc. for $\mathrm{C}_{29} \mathrm{H}_{28} \mathrm{~N}_{6} \mathrm{O}$ (476.23): C 73.09, H 5.92, N 17.63, found: C 73.05, H 5.90, N 17.58.

5-(4-Methylphenyl)-2-phenyl-7-(4-(2-(pyridin-4-yl)ethyl)-1,4-diazepan-1-yl)-oxazolo[4,5-d]pyrimidine (9).

Yield: $82 \%$. mp $135-137{ }^{\circ} \mathrm{C} .{ }^{1} \mathrm{H}-\mathrm{NMR}\left(400 \mathrm{MHz}, \mathrm{DMSO}-d_{6}\right): \delta 8.33(d, J 4.8 \mathrm{~Hz}, 2 \mathrm{H}, \mathrm{HetH}), 8.28-8.25(m, 2 \mathrm{H}$, ArH), $8.21(\mathrm{~d}, J 7.3 \mathrm{~Hz}, 2 \mathrm{H}, \mathrm{ArH}), 7.70-7.62(\mathrm{~m}, 3 \mathrm{H}, \mathrm{ArH}), 7.29(\mathrm{~d}, J 7.7 \mathrm{~Hz}, 2 \mathrm{H}, \mathrm{ArH}), 7.19(d, J 5.0 \mathrm{~Hz}, 2 \mathrm{H}$, $\mathrm{HetH}), 4.07\left(s, 4 \mathrm{H}, \mathrm{CH}_{2}\right), 2.98\left(s, 2 \mathrm{H}, \mathrm{CH}_{2}\right), 2.73\left(s, 6 \mathrm{H}, \mathrm{CH}_{2}\right), 2.37(s, 3 \mathrm{H}, \mathrm{Me}), 1.96\left(s, 2 \mathrm{H}, \mathrm{CH}_{2}\right) .{ }^{13} \mathrm{C}-\mathrm{NMR}(126$ $\left.\mathrm{MHz}, \mathrm{DMSO}-d_{6}\right): \delta 164.4,161.2,159.5,149.5,149.1,148.0,139.6,135.3,132.7,129.4,128.9,127.7,127.6$, 125.8, 124.2, 57.2, 53.7, 32.4, 21.0. MS: $491.0\left([\mathrm{M}+\mathrm{H}]^{+}, \mathrm{C}_{30} \mathrm{H}_{31} \mathrm{~N}_{6} \mathrm{O}^{+}\right.$, calc. 491.3). Anal. calc. for $\mathrm{C}_{30} \mathrm{H}_{30} \mathrm{~N}_{6} \mathrm{O}$ (490.25): C 73.45, H 6.16, N 17.13, found: C 73.42, H 6.15, N 17.20.

General procedure for the synthesis of oxazolo[4,5-d]pyrimidines $\mathbf{1 0 - 1 5}$. To a solution of one of 7piperazinyl- or 7-(1,4-diazepanyl)substituted oxazolo[4,5- $d$ ]pyrimidines $\mathbf{V}(1 \mathrm{mmol})$ and triethylamine (1 mmol) in dioxane $(10 \mathrm{~mL})$ was added one of alkyl(aryl)sulfonyl chlorides $(1 \mathrm{mmol})$. The reaction mixture was heated at $105-110{ }^{\circ} \mathrm{C}$ for $6 \mathrm{~h}$. After removal of the solvent, the residue was triturated with water, filtered off, dried, and recrystallized. 
7-(4-(Methylsulfonyl)piperazin-1-yl)-2-(4-methylphenyl)-5-phenyl-oxazolo[4,5-d]pyrimidine (10). Yield: 75\%. $\mathrm{mp} 289-291^{\circ} \mathrm{C} .{ }^{1} \mathrm{H}-\mathrm{NMR}\left(400 \mathrm{MHz}, \mathrm{CDCl}_{3}\right): \delta 8.50-8.43(\mathrm{~m}, 2 \mathrm{H}, \mathrm{ArH}), 8.12(d, J 8.2 \mathrm{~Hz}, 2 \mathrm{H}, \mathrm{ArH}), 7.49-7.42$ $(m, 3 \mathrm{H}, \mathrm{ArH}), 7.35(d, J 7.8 \mathrm{~Hz}, 2 \mathrm{H}, \mathrm{ArH}), 4.23\left(t, J 5.1 \mathrm{~Hz}, 4 \mathrm{H}, \mathrm{CH}_{2}\right.$ (piperazinyl)), $3.47\left(t, J 5.0 \mathrm{~Hz}, 4 \mathrm{H}, \mathrm{CH}_{2}\right.$ (piperazinyl)), $2.85(d, J 1.6 \mathrm{~Hz}, 3 \mathrm{H}, \mathrm{Me}), 2.46(s, 3 \mathrm{H}, \mathrm{Me}) .{ }^{13} \mathrm{C}-\mathrm{NMR}\left(126 \mathrm{MHz}, \mathrm{CDCl}_{3}\right): \delta 165.7,163.1,156.9$, 147.6, 137.9, 130.0, 128.5, 128.2, 45.8, 34.2, 21.3. MS: $450.0\left([\mathrm{M}+\mathrm{H}]^{+}, \mathrm{C}_{23} \mathrm{H}_{24} \mathrm{~N}_{5} \mathrm{O}_{3} \mathrm{~S}^{+}\right.$, calc. 450.2). Anal. calc. for $\mathrm{C}_{23} \mathrm{H}_{23} \mathrm{~N}_{5} \mathrm{O}_{3} \mathrm{~S}$ (449.15): C 61.45, H 5.16, N 15.58, S 7.13, found: C 61.50, H 5.15, N 15.50, S 7.10.

2-(4-Methylphenyl)-5-phenyl-7-(4-(phenylsulfonyl)piperazin-1-yl)oxazolo[4,5-d]pyrimidine (11). Yield: 73\%. $\mathrm{mp} 247-249{ }^{\circ} \mathrm{C} .{ }^{1} \mathrm{H}-\mathrm{NMR}\left(400 \mathrm{MHz}, \mathrm{CDCl}_{3}\right): \delta 8.43$ (br d, J $\left.6.1 \mathrm{~Hz}, 2 \mathrm{H}, \mathrm{ArH}\right), 8.11(d, J 7.8 \mathrm{~Hz}, 2 \mathrm{H}, \operatorname{ArH}), 7.80(d$, J $7.5 \mathrm{~Hz}, 2 \mathrm{H}, \mathrm{ArH}), 7.60-7.52(m, 3 \mathrm{H}, \mathrm{ArH}), 7.44$ (br s, 3H, ArH), $7.34(d, J 7.7 \mathrm{~Hz}, 2 \mathrm{H}, \operatorname{ArH}), 4.23\left(b r s, 4 \mathrm{H}, \mathrm{CH}_{2}\right.$ (piperazinyl)), 3.25 (br s, 4H, CH (piperazinyl)), 2.46 (s, 3H, Me). ${ }^{13} \mathrm{C}-\mathrm{NMR}\left(126 \mathrm{MHz}^{\mathrm{CDCl}} 3\right): \delta 158.2,143.9$, 135.6, 133.4, 130.6, 130.0, 129.5, 128.6, 128.5, 128.3, 127.9, 46.1, 44.8, 22.0. MS: $512.0\left([M+]^{+}\right.$, $\mathrm{C}_{28} \mathrm{H}_{26} \mathrm{~N}_{5} \mathrm{O}_{3} \mathrm{~S}^{+}$, calc. 512.2). Anal. calc. for $\mathrm{C}_{28} \mathrm{H}_{25} \mathrm{~N}_{5} \mathrm{O}_{3} \mathrm{~S}$ (511.17): C 65.74, $\mathrm{H} \mathrm{4.93,} \mathrm{N} \mathrm{13.69,} \mathrm{S} \mathrm{6.27,} \mathrm{found:} \mathrm{C}$ 65.70, H 4.91, N 13.70, S 6.25 .

2-(4-Methylphenyl)-5-phenyl-7-(4-tosylpiperazin-1-yl)oxazolo[4,5-d]pyrimidine (12). Yield: $72 \%$. mp 261 $263{ }^{\circ} \mathrm{C} .{ }^{1} \mathrm{H}-\mathrm{NMR}\left(400 \mathrm{MHz}, \mathrm{CDCl}_{3}\right): \delta 8.46-8.39(\mathrm{~m}, 2 \mathrm{H}, \mathrm{ArH}), 8.09(d, J 7.9 \mathrm{~Hz}, 2 \mathrm{H}, \mathrm{ArH}), 7.66(d, J 8.3 \mathrm{~Hz}, 2 \mathrm{H}$, ArH), $7.46-7.40(m, 3 \mathrm{H}, \mathrm{ArH}), 7.32(t, J 7.8 \mathrm{~Hz}, 4 \mathrm{H}, \mathrm{ArH}), 4.20\left(t, J 5.0 \mathrm{~Hz}, 4 \mathrm{H}, \mathrm{CH}_{2}\right.$ (piperazinyl)), $3.21(t, J 5.0$ $\mathrm{Hz}, 4 \mathrm{H}, \mathrm{CH}_{2}$ (piperazinyl)), 2.45 (s, 3H, Me), 2.39 (s, 3H, Me). ${ }^{13} \mathrm{C}-\mathrm{NMR}\left(126 \mathrm{MHz}, \mathrm{CDCl}_{3}\right): \delta 165.6,163.2,160.7$, 147.4, 144.3, 143.7, 137.9, 132.4, 130.3, 130.0, 129.9, 128.4, 128.3, 128.2, 127.9, 127.8, 123.3, 46.1, 44.6, 21.9, 21.7. MS: $526.2\left([\mathrm{M}+\mathrm{H}]^{+}, \mathrm{C}_{29} \mathrm{H}_{28} \mathrm{~N}_{5} \mathrm{O}_{3} \mathrm{~S}^{+}\right.$, calc. 526.2). Anal. calc. for $\mathrm{C}_{29} \mathrm{H}_{27} \mathrm{~N}_{5} \mathrm{O}_{3} \mathrm{~S}$ (525.18): C 66.27, $\mathrm{H}$ 5.18, N 13.32, S 6.10, found: C 66.25, H 5.15, N 13.24, S 6.20.

7-(4-(Methylsulfonyl)piperazin-1-yl)-2,5-diphenyl-oxazolo[4,5-d]pyrimidine (13). Yield: 71\%. mp $275-277^{\circ} \mathrm{C}$. ${ }^{1} \mathrm{H}-\mathrm{NMR}\left(400 \mathrm{MHz}, \mathrm{CDCl}_{3}\right): \delta 8.51-8.44(\mathrm{~m}, 2 \mathrm{H}, \mathrm{ArH}), 8.29-8.22(\mathrm{~m}, 2 \mathrm{H}, \mathrm{ArH}), 7.66-7.52(\mathrm{~m}, 3 \mathrm{H}, \mathrm{ArH}), 7.50-$ $7.43(\mathrm{~m}, 3 \mathrm{H}, \mathrm{ArH}), 4.26\left(\mathrm{t}, J 5.1 \mathrm{~Hz}, 4 \mathrm{H}, \mathrm{CH}_{2}\right.$ (piperazinyl)), $3.48\left(\mathrm{t}, J 5.1 \mathrm{~Hz}, 4 \mathrm{H}, \mathrm{CH}_{2}\right.$ (piperazinyl)), 2.85 (s, 3H, Me). ${ }^{13} \mathrm{C}-\mathrm{NMR}\left(151 \mathrm{MHz}, \mathrm{CDCl}_{3}\right): \delta 164.3,162.0,160.1,153.2,143.6,136.9,132.0,129.5,128.3,127.5,127.2$, 126.7, 125.1, 44.8, 33.9. MS: $436.0\left([\mathrm{M}+\mathrm{H}]^{+}, \mathrm{C}_{22} \mathrm{H}_{22} \mathrm{~N}_{5} \mathrm{O}_{3} \mathrm{~S}^{+}\right.$, calc. 436.1). Anal. calc. for $\mathrm{C}_{22} \mathrm{H}_{21} \mathrm{~N}_{5} \mathrm{O}_{3} \mathrm{~S}$ (435.14): C 60.68, H 4.86, N 16.08, S 7.36, found: C 60.65, H 4.86, N 16.00, S 7.30.

2-(4-Methylphenyl)-5-phenyl-7-(4-(phenylsulfonyl)-1,4-diazepan-1-yl)-oxazolo[4,5-d]pyrimidine (14). Yield: 74\%. mp $229-231{ }^{\circ} \mathrm{C} .{ }^{1} \mathrm{H}-\mathrm{NMR}\left(400 \mathrm{MHz}, \mathrm{DMSO}-d_{6}\right): \delta 8.34-8.31(m, 2 \mathrm{H}, \mathrm{ArH}), 8.04(d, J 7.8 \mathrm{~Hz}, 2 \mathrm{H}, \mathrm{ArH})$, $7.68(d, J 7.6 \mathrm{~Hz}, 2 \mathrm{H}, \mathrm{ArH}), 7.49-7.36(m, 8 \mathrm{H}, \mathrm{ArH}), 4.09\left(t, J 5.6 \mathrm{~Hz}, 2 \mathrm{H}, \mathrm{CH}_{2}\right.$ (diazepanyl)), $3.98(t, J 5.6 \mathrm{~Hz}, 2 \mathrm{H}$, $\mathrm{CH}_{2}$ (diazepanyl)), $3.61\left(t, J 5.6 \mathrm{~Hz}, 2 \mathrm{H}, \mathrm{CH}_{2}\right.$ (diazepanyl)), $3.41\left(\right.$ br s, 2H, $\mathrm{CH}_{2}$ (diazepanyl)), $2.39(s, 3 \mathrm{H}, \mathrm{Me})$,

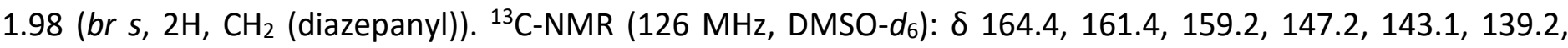
137.9, 132.6, 130.0, 129.9, 129.1, 128.3, 127.7, 127.6, 127.4, 126.4, 122.9, 46.9, 21.3. MS: $526.2\left([M+]^{+}\right.$, $\mathrm{C}_{29} \mathrm{H}_{28} \mathrm{~N}_{5} \mathrm{O}_{3} \mathrm{~S}^{+}$, calc. 526.2). Anal. calc. for $\mathrm{C}_{29} \mathrm{H}_{27} \mathrm{~N}_{5} \mathrm{O}_{3} \mathrm{~S}$ (525.18): C 66.27, H 5.18, N 13.32, S 6.10, found: C 66.31, H 5.20, N 13.36, S 6.15 .

2-(4-Methylphenyl)-5-phenyl-7-(4-tosyl-1,4-diazepan-1-yl)oxazolo[4,5-d]pyrimidine (15). Yield: $77 \%$. mp 192 - $194{ }^{\circ} \mathrm{C} .{ }^{1} \mathrm{H}$ NMR $\left(400 \mathrm{MHz}, \mathrm{CDCl}_{3}\right): \delta 8.44(d, J 6.0 \mathrm{~Hz}, 2 \mathrm{H}, \mathrm{ArH}), 8.10(d, J 7.9 \mathrm{~Hz}, 2 \mathrm{H}, \mathrm{ArH}), 7.55(d, J 8.0 \mathrm{~Hz}$, $2 \mathrm{H}, \mathrm{ArH}), 7.46-7.44(m, 3 \mathrm{H}, \mathrm{ArH}), 7.36(d, J 8.0 \mathrm{~Hz}, 2 \mathrm{H}, \mathrm{ArH}), 7.04(d, J 7.9 \mathrm{~Hz}, 2 \mathrm{H}, \mathrm{ArH}), 4.18(t, J 5.5 \mathrm{~Hz}, 2 \mathrm{H}$, $\mathrm{CH}_{2}$ (diazepanyl)), $4.10\left(t, J 6.3 \mathrm{~Hz}, 2 \mathrm{H}, \mathrm{CH}_{2}\right.$ (diazepanyl)), $3.62\left(t, J 5.6 \mathrm{~Hz}, 2 \mathrm{H}, \mathrm{CH}_{2}\right.$ (diazepanyl)), $3.43(t, J 6.0$ $\mathrm{Hz}, 2 \mathrm{H}, \mathrm{CH}_{2}$ (diazepanyl)), $2.47(s, 3 \mathrm{H}, \mathrm{Me}), 2.27(s, 3 \mathrm{H}, \mathrm{Me}), 2.16\left(t, J 6.6 \mathrm{~Hz}, 2 \mathrm{H}, \mathrm{CH}_{2}\right.$ (diazepanyl)). ${ }^{13} \mathrm{C}-\mathrm{NMR}$ $\left(126 \mathrm{MHz}_{1} \mathrm{CDCl}_{3}\right): \delta 160.7,143.6,138.1,130.2,130.0,129.7,128.4,128.1,126.8,123.6,47.5,21.9,21.5$. MS: $540.2\left([\mathrm{M}+\mathrm{H}]^{+}, \mathrm{C}_{30} \mathrm{H}_{30} \mathrm{~N}_{5} \mathrm{O}_{3} \mathrm{~S}^{+}\right.$, calc. 540.2). Anal. calc. for $\mathrm{C}_{30} \mathrm{H}_{29} \mathrm{~N}_{5} \mathrm{O}_{3} \mathrm{~S}$ (539.20): C 66.77, H 5.42, N 12.98, S 5.94, found: C 66.75, H 5.40, N 13.05, S 5.90. 


\section{Biology}

Antiviral and cytotoxicity assays. The antiviral activity screening of the compounds was performed at the University of Alabama at Birmingham. The antiviral properties of the synthesized compounds were studied against four DNA viruses belonging to the families Herpesviridae (HSV-1, HCMV, and VZV) and Polyomaviridae (BKV). Evaluation of activity was performed in 384 well plates by methods previously reported ${ }^{13-16}$ Briefly, drugs were diluted in 384 well plates with cells seeded prior to, or added after, the dilutions. Drug and control concentration ranged from 0.048 to $150 \mu \mathrm{M}$, vehicle was DMSO. Virus was then added at the appropriate MOI (for antiviral assessment) or media (for cytotoxic assessment) and the plates incubated for $2-14$ days $\left(37^{\circ} \mathrm{C}\right.$, $5 \%\left(\mathrm{CO}_{2}\right)$, according to the virus used. For endpoint determinations, the plates were harvested/treated as indicated (Table 3). Results for tested compound were reported as $\mathrm{EC}_{50}$ and $\mathrm{CC}_{50}$ that are of concentrations reducing viral replication and cell viability by $50 \%$ with respect to the untreated control. $\mathrm{SI}_{50}$ value is a selectivity index calculated as a ratio of $\mathrm{CC}_{50} / \mathrm{EC}_{50}$.

Table 3. Viruses and assays used for determination of antiviral activity and cytotoxicity of synthesized compounds (cell line HFF)

\begin{tabular}{cc}
\hline Virus (Strain) & Control Assay Name \\
\hline Herpes simplex virus 1 (E-377) & CellTiter-Glo (Cytopathic effect/Toxicity) \\
Human cytomegalovirus (AD169) & CellTiter-Glo (Cytopathic effect/Toxicity) \\
Varicella-Zoster virus (Ellen) & CellTiter-Glo (Cytopathic effect/Toxicity) \\
BK polyomavirus (Gardner) & Quantitative polymerase chain reaction \\
& (DNA)/CellTiter-Glo (Toxicity) \\
\hline
\end{tabular}

\section{Acknowledgements}

We would like to thank Enamine Ltd for the material and technical support. These studies were funded in whole or in part with Federal funds from the National Institute of Allergy and Infectious Diseases, National Institutes of Health, Department of Health and Human Services, under Contract Nos. HHSN272201100016I (MNP) and HHSN75N93019D00016 (SHJ). We thank the National Research Foundation of Ukraine (2020.01/0075 NRFU competition "Science for the Security of Human and Society") for financial support.

\section{Supplementary Material}

Copies of the ${ }^{1} \mathrm{H}$ NMR, ${ }^{13} \mathrm{C}$ NMR and LCMS spectra as well as ADMET properties of compounds 1-15 predicted by pkCSM online server (Table S1) are given in the Supplementary Material file associated with this manuscript. 


\section{References}

1. De Coen, L.; Roman, B.; Movsisyan, M.; Heugebaert, T.; Stevens, C. Eur. J. Org. Chem. 2018, 2018, 21482166.

https://doi.org/10.1002/ejoc.201800133

2. Zhirnov, V.V.; Velihina, Ye.S.; Mitiukhin, O.P.; Brovarets, V.S. Chem. Biol. Drug Des. 2021, 98, 561-581. https://doi.org/10.1111/cbdd.13911

3. Myllymäki, M.J.; Käsnänen, H.; Kataja, A.O.; Lahtela-Kakkonen, M.; Saario, S.M.; Poso, A.; Koskinen, A.M. Eur. J. Med. Chem. 2009, 44, 4179-4191.

https://doi.org/10.1016/i.ejmech.2009.05.012

4. Velihina, Ye.S.; Kachaeva, M.V.; Pilyo, S.G.; Zhirnov, V.V.; Brovarets, V.S. Der Pharma Chem. 2018, $10,1-10$.

5. Velihina, Ye.S.; Kachaeva, M.V.; Pilyo, S.G.; Mitiukhin, O.P.; Zhirnov, V.V.; Brovarets, V.S. Chem. R.J. 2018, 3, 81-93.

6. Velihina, Ye.; Scattolin, T.; Bondar, D.; Pil’o, S.; Obernikhina, N.; Kachkovskyi, O.; Semenyuta, I.; Caligiuri, I.; Rizzolio, F.; Brovarets, V.; Karpichev, Y.; Nolan, S.P. Helv. Chim. Acta 2020, 103, e2000169. https://doi.org/10.1002/hlca.202000169

7. Kumar, S.; Deep, A.; Narasimhan, B. Curr. Bioact. Compd. 2019, 15, 289-303. https://doi.org/10.2174/1573407214666180124160405

8. Sochacka-Ćwikła, A.; Regiec, A.; Zimecki, M.; Artym, J.; Zaczyńska, E.; Kocięba, M.; Kochanowska, I; Bryndal, I; Pyra, A.; Mączyński, M. Molecules 2020, 25, 3558.

https://doi.org/10.3390/molecules25153558

9. Kachaeva, M.; Pilyo, S.; Kornienko, A.; Prokopenko, V.; Zhirnov, V.; Prichard, M.; Keith, K.; Yang, G.; Wang, H.; Banerjee, N.; Chow, L.; Broker, T.; Brovarets, V. Ibnosina J. Med. Biomed. Sci. 2017, 9, 111-118.

10. Drach, B.S.; Miskevich, G.N. Russ. J. Organ. Chem. 1974, 10, 2315 (Chem. Abstr., 1975, 82, 72843).

11. Lipinski, C.A.; Lombardo, F.; Dominy, B.W.; Feeney, P.J. Adv. Drug Deliv. Rev. 1997, 23, 3-25. https://doi.org/10.1016/S0169-409X(96)00423-1

12. Pires, D.E.V.; Blundell, T.L.; Ascher, D.B. J. Med. Chem. 2015, 58, 4066-4072. https://doi.org/10.1021/acs.jmedchem.5b00104

13. Sidwell, R.W.; Smee, D.F. Antiviral Res. 2000, 48, 1-16. https://doi.org/10.1016/S0166-3542(00)00125-X

14. Hartline, C.B.; Keith, K.A.; Eagar, J.; Harden, E.A.; Bowlin, T.L.; Prichard, M.N. Antiviral Res. 2018, 159, 104112.

https://doi.org/10.1016/i.antiviral.2018.09.015

15. Keith, K.A.; Hartline, C.B.; Bowlin, T.L.; Prichard, M.N. Antiviral Res. 2018, 159, 122-129. https://doi.org/10.1016/i.antiviral.2018.09.016

16. Beadle, J.R.; Valiaeva, N.; Yang, G.; Yu, J.H.; Broker, T.R.; Aldern, K.A.; Harden, E.A.; Keith, K.A.; Prichard, M.N.; Hartman, T.; Buckheit, R.W. Jr.; Chow, L.T.; Hostetler, K.Y. J. Med. Chem. 2016, 59, 10470-10478. https://doi.org/10.1021/acs.jmedchem.6b00659 